\title{
High-field magnetocrystalline anisotropic resistance effect in (Ga,Mn)As
}

\author{
D. Wu, ${ }^{1,2}$ Peng Wei, ${ }^{1}$ E. Johnston-Halperin, ${ }^{3}$ D. D. Awschalom, ${ }^{4}$ and Jing Shi ${ }^{1}$ \\ ${ }^{1}$ Department of Physics and Astronomy, University of California, Riverside, California 92521, USA \\ ${ }^{2}$ National Laboratory of Solid State Microstructures and Department of Physics, Nanjing University, 22 Hankou Road, Nanjing 210093, \\ China \\ ${ }^{3}$ Department of Physics, Ohio State University, Columbus, Ohio 43210, USA \\ ${ }^{4}$ Department of Physics, University of California, Santa Barbara, California 93106, USA
}

(Received 25 October 2007; revised manuscript received 19 December 2007; published 17 March 2008)

\begin{abstract}
As the magnetization rotates in the (001) plane of epitaxial $(\mathrm{Ga}, \mathrm{Mn})$ As films, we observe both two- and fourfold oscillations of comparable magnitude in the longitudinal resistivity. This behavior is different from the usual anisotropic magnetoresistance effect in polycrystalline films. The fourfold or cubic symmetry vanishes at the Curie temperature $T_{C}$, indicating that it originates from the long-range ferromagnetic phase in single crystal films. In contrast, the twofold symmetry persists above $T_{C}$, suggesting its origin to be from the alignment of spins with random orientations. However, the transverse, or planar Hall, resistivity only contains a twofold oscillation. The temperature dependence of the magnetocrystalline anisotropic resistance effect is explained by a two-component model.
\end{abstract}

DOI: 10.1103/PhysRevB.77.125320

PACS number(s): 73.50.- h, 75.47.- m, 75.50.Pp

The anisotropic magnetoresistance (AMR) effect in ferromagnetic (FM) materials arises from the difference in the scattering rate when the magnetization direction is oriented parallel or perpendicular to an electrical current. ${ }^{1}$ It is a convenient tool often used for characterizing the state of the magnetization in ferromagnetic metals ${ }^{2}$ and semiconductors. ${ }^{3,4}$ In ferromagnetic semiconductors such as GaMnAs, AMR has been linked to the intrinsic band structure properties in the presence of spin-orbit interaction. ${ }^{5}$ For polycrystalline materials, the resistivity typically shows a twofold symmetry because the magnetocrystalline effect in the randomly oriented grains is averaged out. In single crystal films, however, also due to the spin-orbit interaction, AMR may contain higher-order terms that reflect the symmetry of the crystals. ${ }^{6}$ Nevertheless, in many cases, the highorder terms are small and often neglected. ${ }^{7,8}$ Here, we have conducted a series of experiments in epitaxially grown GaMnAs films and found a very strong fourfold magnetoresistance effect that is correlated with the crystalline symmetry of the films. This fourfold term is found to be connected to the long-range FM order below $T_{C}$; therefore, its temperature and field dependences reveal valuable information about the ferromagnetic ordering in the materials.

We studied two $\mathrm{Ga}_{1-x} \mathrm{Mn}_{x}$ As samples with $x=0.039$ and 0.059 which were grown on $\mathrm{GaAs}(001)$ wafers at $250{ }^{\circ} \mathrm{C}$ by molecular beam epitaxy in an As-rich environment. The samples were patterned into the standard Hall bar using photolithography and wet chemical etching. For resistivity measurements, the current flows along a [110] direction, as schematically shown in Fig. 1(a), and both the longitudinal and transverse or the planar Hall dc resistivities $\rho_{x x}$ and $\rho_{x y}$ are measured simultaneously using the four-probe method. The measurements are performed in a superconducting magnet equipped with a rotating sample holder, which allows us to continuously change the angle $\phi$ between the magnetic field $H$ and the electric current $I$, as illustrated in Fig. 1(a). As the sample is rotated around [001], $H$ always lies in the sample plane, and both $\rho_{x x}$ and $\rho_{x y}$ are recorded as angle $\phi$ is varied. The angular dependence is taken for different magnetic field strengths and temperatures. Since both samples show similar behaviors in their angular dependence, we will focus our discussions on the $x=0.059$ sample below.

$\rho_{x x}$ as a function of temperature at $H=0$ for $\mathrm{Ga}_{0.941} \mathrm{Mn}_{0.059}$ As shows a peak due to the spin disorder scattering enhanced resistivity, ${ }^{9}$ as shown in the inset of Fig. 1(b). From $\rho_{x x}$ vs $T$, we can estimate the Curie temperature, $T_{C} \sim 49 \mathrm{~K}$, which agrees with the $T_{C}$ found from the superconducting quantum interference device measurements. Figure 1(b) shows $\rho_{x x}$ as a function of $H$ for the same sample at

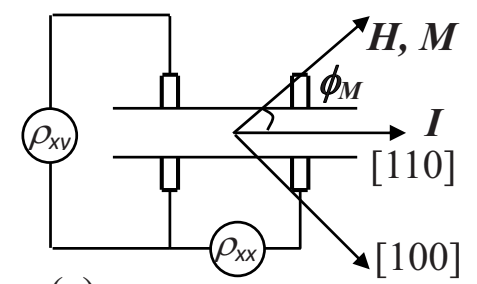

(a)

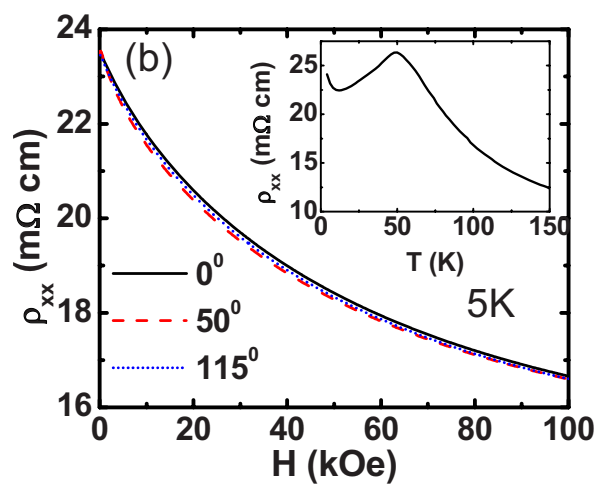

FIG. 1. (Color online) (a) Sketch of experimental geometry. Current $I$ flows along [110] and magnetization $\mathbf{M}$ is aligned with the external field $\mathbf{H}$ which rotates in the (001) plane. (b) Longitudinal resistivity as a function of in-plane field at different field orientations, $\phi=0^{\circ}, 50^{\circ}$, and $115^{\circ}$, at $5 \mathrm{~K}$ for $\mathrm{Ga}_{0.941} \mathrm{Mn}_{0.059} \mathrm{As}$. The inset shows the resistivity as a function of temperature at zero field. 


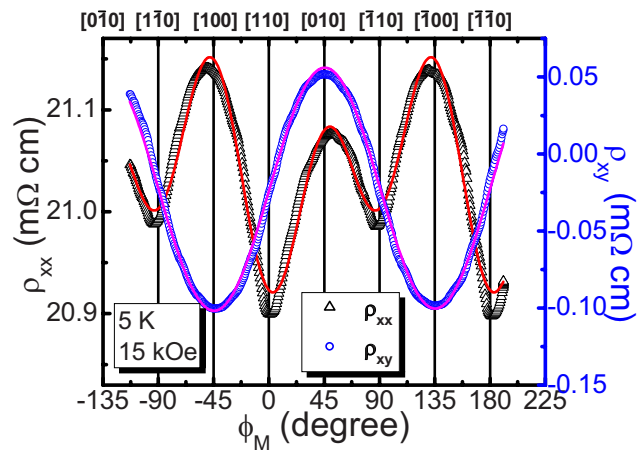

FIG. 2. (Color online) Angular dependence of the longitudinal resistivity $\rho_{x x}$ and planar Hall resistivity $\rho_{x y}$ at $H=15 \mathrm{kOe}$ and $T$ $=5 \mathrm{~K}$ for $\mathrm{Ga}_{0.941} \mathrm{Mn}_{0.059} \mathrm{As}$. The solid lines are the fits.

$5 \mathrm{~K}$ with $H$ applied at three different angles with current $I$. In this field range, $\rho_{x x}$ exhibits negative magnetoresistance (MR), which is in agreement with what was reported previously. ${ }^{10}$ This negative MR has been attributed to the scattering of carriers off paramagnetic $\mathrm{Mn}^{2+}$ ions or magnetic boundary polarons, ${ }^{11,12}$ or attributed to the weak localization effect. ${ }^{13}$ Since $H$ far exceeds the coercive field $H_{c}(<50 \mathrm{Oe}$ at $5 \mathrm{~K})$ or the anisotropy field $H_{k}(\sim 1000 \mathrm{Oe})$, the FM magnetization $\mathbf{M}$ should be fully aligned with $H$. As seen in Fig. 1(b), this high-field MR actually depends on the direction of $\mathbf{H}$ or $\mathbf{M}$.

The anisotropy of this high-field MR can be clearly seen as the sample is continuously rotated in a constant in-plane field whose strength is greater than that of the anisotropy field. At such a high field, $\phi_{M}$, the angle between $\mathbf{M}$ and $I$, should be nearly identical to $\phi$, the angle between $\mathbf{H}$ and $I$. In Fig. 2, we plot both $\rho_{x x}$ and $\rho_{x y}$ at $5 \mathrm{~K}$ with $H=15 \mathrm{kOe}$. Both $\rho_{x x}$ and $\rho_{x y}$ oscillate as the sample is rotated about [001], but the oscillation periods are different. Apparently, the oscillations in $\rho_{x x}$ cannot be described by a simple $\cos 2 \phi_{M}$ function that is normally expected for polycrystalline FM films. There is a large fourfold or cubic component present which peaks at approximately $-45^{\circ}, 45^{\circ}$, and $135^{\circ}$ in $\phi_{M}$. In GaMnAs (001) epitaxial films, this cubic symmetry simply coincides with the fourfold symmetry of the (001) plane of the zinc-blende crystalline structure. To include both types of symmetry, we use $\rho_{x x}=\rho_{0}\left(1+A_{u} \cos 2 \phi_{M}\right.$ $\left.+A_{c} \cos 4 \phi_{M}\right)$ to fit our data and obtain the relative oscillation amplitudes for $\cos 2 \phi_{M}$ and $\cos 4 \phi_{M}$ terms, i.e., $A_{u}$ $=0.25 \%$ and $A_{c}=0.37 \%$, respectively. The fourfold anisotropy is actually stronger than the twofold anisotropy in this case. By the conventional definition $\mathrm{AMR}_{\mathrm{ip}} \equiv\left[\rho_{J|| M}\right.$ $\left.-\rho_{J \perp M}\right] / \rho_{J \perp M}$, the in-plane AMR ratio is calculated to be about $-0.41 \%$, and the sign agrees with the theoretical prediction. ${ }^{5,14}$ Unlike the symmetry in $\rho_{x x}$, the planar Hall resistivity $\rho_{x y}$ only contains a twofold oscillation, as shown in Fig. 2, and it can be fitted well with a single $\sin 2 \phi_{M}$ function. Note that $\rho_{x y}$ has a nonzero offset, which is likely caused by a small $\rho_{x x}$ component due to imperfect alignment between the two transverse leads.

For polycrystalline or isotropic ferromagnetic materials, $\rho_{x x}$ and $\rho_{x y}$ both depend on the relative angle $\phi_{M}$ between magnetization $M$ and current $I$. The AMR in single domain model can be described by two equations: ${ }^{1}$

$$
\begin{gathered}
\rho_{x x}=\rho_{\perp}+\left(\rho_{\|}-\rho_{\perp}\right) \cos ^{2} \phi_{M}, \\
\rho_{x y}=\left(\rho_{\|}-\rho_{\perp}\right) \sin \phi_{M} \cos \phi_{M},
\end{gathered}
$$

where $\rho_{\perp}$ and $\rho_{\|}$are the resistivities for $M \perp I$ and $M \| I$, respectively. Both $\rho_{x x}$ and $\rho_{x y}$ have a twofold or uniaxial symmetry about the current direction with the rotation of $M$ and are intimately related to each other. The equations have been adopted to explain AMR observed in GaMnAs films. ${ }^{7,15,16}$ However, this picture is not applicable here since there is an additional strong fourfold term in $\rho_{x x}$. To see how different anisotropy terms contribute to $\rho_{x x}$ and $\rho_{x y}$ in single crystal films, here, we use a phenomenological description for the anisotropic transport. We assume that the resistivity contains up to the fourth order terms in direction cosines $\alpha$

$$
\begin{aligned}
\rho_{i j}= & a_{i j}+a_{k i j} \alpha_{k}+a_{k l i j} \alpha_{k} \alpha_{l}+a_{k l m i j} \alpha_{k} \alpha_{l} \alpha_{m}+a_{k l m n i j} \alpha_{k} \alpha_{l} \alpha_{m} \alpha_{n} \\
& +\cdots
\end{aligned}
$$

where $\alpha$ is the direction cosines of $\mathbf{M}$ with the cubic axes, and $a_{i j}, a_{k i j}, a_{k l m i j}$, and $a_{k l m n i j}$ are the elements of the resistivity tensor of various orders. $i$ and $j$ can be in any of the three orthogonal directions, but in the context of our discussions, they are either along or perpendicular to the current in the film plane. The resistivity tensor $\rho_{i j}$ then relates to AMR effect and the planar Hall effect through Ohm's law: $E_{i}$ $=\rho_{i j}(\alpha) J_{j}$, where $E_{i}$ is the electric field along $i$ and $J_{j}$ is the current density along $j$. For instance, if a current $J_{x}$ flows along the $x$ axis, the electric field $E_{x}$ in this direction depends on the direction cosines of $\mathbf{M}$ with respect to the cubic axes via $E_{x}=\rho_{x x}(\alpha) J_{x}$; for the same current density, it also induces an electric field $E_{y}$ or a finite voltage along the $y$ axis if $\rho_{y x}$ is not zero. The latter effect is the planar Hall effect due to an in-plane magnetization and anisotropic resistivity. It has a completely different physical origin from that of the regular Hall effect which is caused by the Lorentz force from a perpendicular magnetic field.

Many of the matrix elements actually vanish due to the symmetry of the cubic crystal and the Onsager relation. ${ }^{17}$ If we apply this equation to our device geometry, where the voltages along [110] and [-110] are measured with a current flowing along [110], then,

$$
\begin{aligned}
\rho_{x x}= & E_{x x} / J=C_{0}+1 / 2 C_{1}+3 / 8 C_{2}+1 / 2 C_{4} \cos 2 \phi_{M} \\
& -1 / 8 C_{2} \cos 4 \phi_{M}=\rho_{0}\left(1+A_{u} \cos 2 \phi_{M}+A_{c} \cos 4 \phi_{M}\right),
\end{aligned}
$$

$$
\rho_{x y}=E_{x y} / J=1 / 2\left(C_{1}+C_{2}\right) \sin 2 \phi_{M}=\rho_{1} \sin 2 \phi_{M},
$$

where $C_{0}, C_{1}, C_{2}$, and $C_{4}$ are a set of constants that is related to the coefficients in Eq. (3) by

$$
\begin{gathered}
C_{0}=a_{11}+a_{1122}+a_{111122}, \quad C_{1}=a_{1111}-a_{1122}-2 a_{112211}, \\
C_{2}=a_{111111}+2 a_{111122}-a_{112211}, \\
C_{4}=a_{2323}+a_{111212} .
\end{gathered}
$$

These four constants can be determined from $A_{u}, A_{c}, \rho_{0}$, and $\rho_{1}$, which can be obtained by fitting the experimental data. As a direct consequence of the crystal symmetry, $\rho_{x x}$ contains 

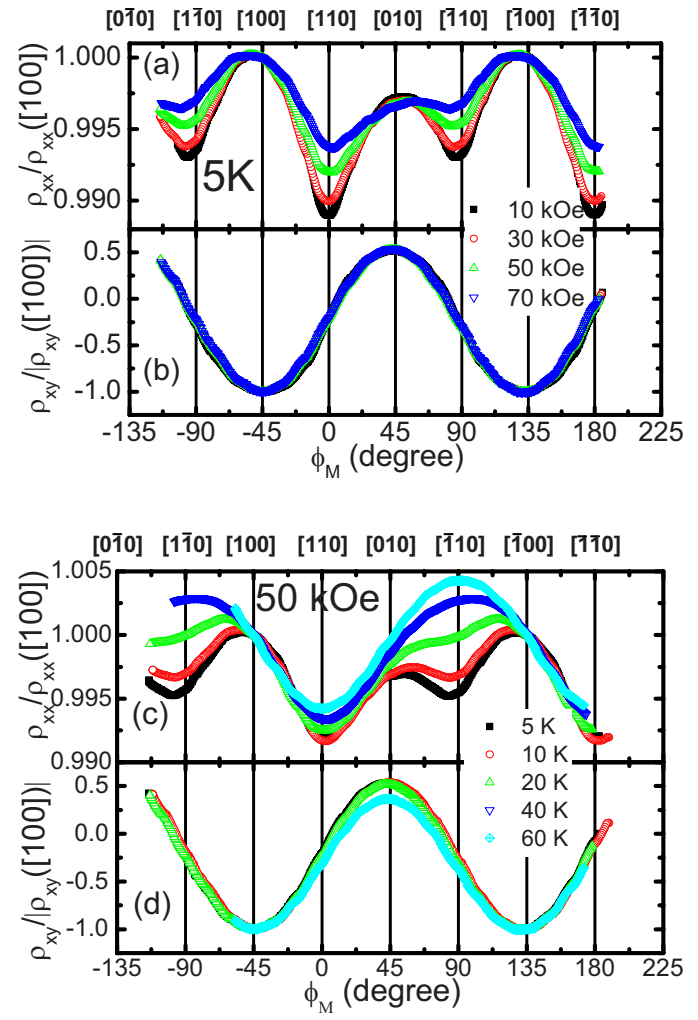

FIG. 3. (Color online) (a) $\rho_{x x}$ and (b) $\rho_{x y}$ at a set of fields for $T=5 \mathrm{~K}$; (c) $\rho_{x x}$ and (d) $\rho_{x y}$ at different temperatures for a fix field $H=50$ kOe. $\rho_{x x}$ and $\rho_{x y}$ are normalized to the resistivity value at $M \|[100]$.

both cubic and uniaxial symmetry terms, whereas $\rho_{x y}$ contains only the uniaxial symmetry term in Eqs. (4) and (5), which agrees well with our experiments.

Now, let us examine how these two anisotropy terms depend on the magnetic field and temperature. Figures 3(a) and 3(b) show $\rho_{x x}$ and $\rho_{x y}$ vs $\phi_{M}$ for a series of fields $H=10,30$, 50 , and $70 \mathrm{kOe}$ measured at $5 \mathrm{~K}$. The $y$ axis shows the scaled resistivity normalized to the resistivity value when $H$ is parallel to the [100] direction. Clearly, both uniaxial and cubic anisotropies in $\rho_{x x}$ coexist at all $H$, but the oscillation amplitude steadily decreases at higher fields. Note that the total resistivity also decreases; therefore, the absolute resistance swing decreases even more at high fields. By decomposing the total resistivity into the cubic and uniaxial terms, we find that $A_{c}$ decays much faster than $A_{u}$ as $H$ increases. In contrast, the ratio $\rho_{x y} / \rho_{x y}[100]$ does not change within experiment uncertainty since all $\rho_{x y} / \rho_{x y}[100]$ curves fall on top of each other for fields up to $70 \mathrm{kOe}$. No measurable cubic symmetry is present at these fields.

At a fixed field of $50 \mathrm{kOe}, \rho_{x x}$ and $\rho_{x y}$ are displayed for different temperatures in Figs. 3(c) and 3(d). An obvious feature is that the fourfold oscillation steadily diminishes as the temperature increases, and it disappears around $T_{C}$. This strongly suggests that the cubic symmetry originates from the long-rang FM order. In contrast, the twofold oscillation persists even above $T_{C}$ where the FM order no longer exists. However, both the symmetry and amplitude of $\rho_{x y} / \rho_{x y}[100]$ remain unchanged below $T_{C}$ and only show weak temperature dependence above $T_{C}$.
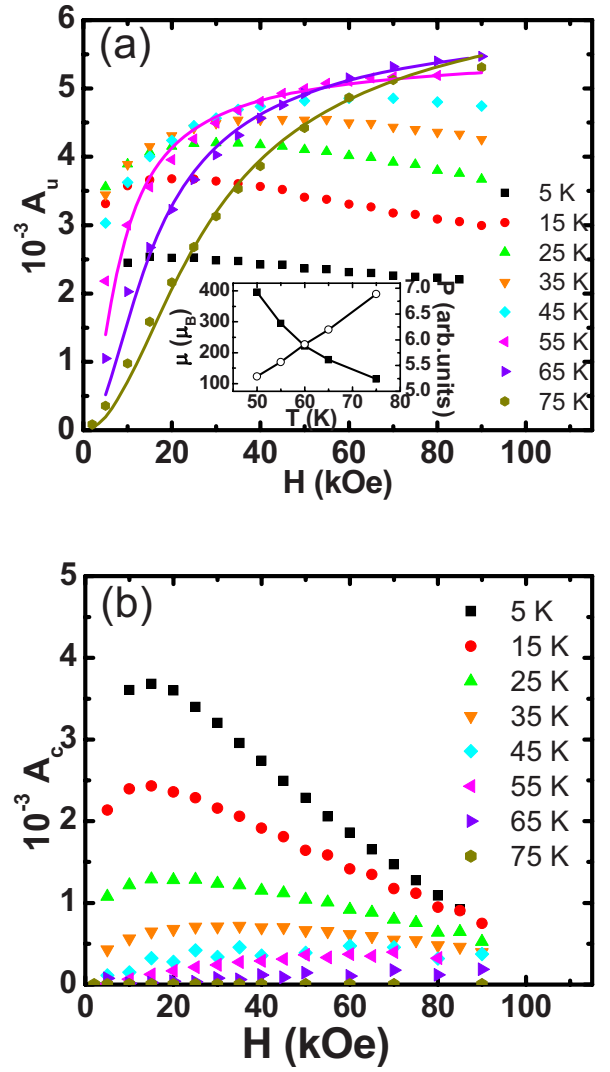

FIG. 4. (Color online) Magnetic field dependence of coefficients (a) $A_{u}$ and (b) $A_{c}$ for $\mathrm{Ga}_{0.0941} \mathrm{Mn}_{0.059} \mathrm{As}$, obtained from fitting. The inset of (a) shows the magnetic moment of each cluster (filled square) and the contribution from all clusters, $P$ (open circle), to $A_{u}$. The solid line in (a) is the fit for $T>T_{C}$, described in the text.

In nonmagnetic crystals, MR can show different behaviors when a current flows along two orthogonal crystallographic directions. In FM crystals, this MR anisotropy adds to the usual AMR which is sensitive to the direction of $\mathbf{M}$. This MR and its anisotropy have been attributed to the Lorentz force and follow Kohler's rule. ${ }^{18}$ The Lorentz-force induced MR or intrinsic MR is always positive. However, the MR effect observed in our experiments clearly does not share the same origin with the intrinsic MR since ours is always negative, as shown in Fig. 1(b). Moreover, it exhibits a more complex temperature dependence [Fig. 4(a)]. Although the negative MR was previously attributed to the weak localization effect, ${ }^{12}$ it cannot easily explain why it depends on the crystal orientations and the relative direction between the current and the magnetic field.

The extracted coefficients $A_{u}$ and $A_{c}$ are plotted as functions of the magnetic field at various temperatures in Fig. 4 and also replotted as functions of the temperature for different fields in Fig. 5. $A_{c}$ shows a monotonic and drastic decrease for all magnetic fields as $T$ approaches $T_{C}$ from below, indicating that the cubic symmetry is induced by the FM phase. A similar result has been also reported recently. ${ }^{19}$ In contrast, $A_{u}$ shows nonmonotonic temperature dependence at those fields. In order to explain this complex temperature dependence of the twofold symmetry, we propose the following two-component model. We assume that at $T<T_{C}$, in ad- 


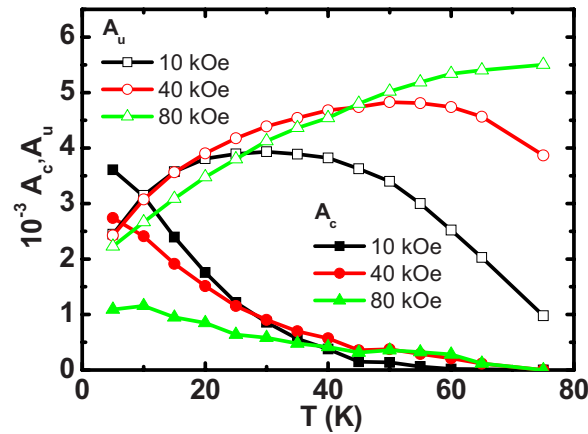

FIG. 5. (Color online) Temperature dependence of coefficients $A_{u}$ (open symbols) and $A_{c}$ (solid symbols) for $\mathrm{Ga}_{0.0941} \mathrm{Mn}_{0.059} \mathrm{As}$ at 10, 40, and $80 \mathrm{kOe}$, obtained from fitting using Eq. (4).

dition to a long-range FM component, there exists a second component that is comprised of individual magnetic clusters. One realization of this two-component picture could be a magnetic polaron. More explicitly, since the FM coupling between Mn ions $(S=5 / 2)$ is mediated through the delocalized holes in GaMnAs, each $\mathrm{Mn}^{2+}$ ion and its neighboring free holes behave like a magnetic cluster, known as a bound magnetic polaron (BMP). This carrier-mediated interaction distinguishes the ferromagnetic semiconductors from the conventional ferromagnets where the Heisenberg direct exchange links the adjacent spins. As the temperature is lowered below $T_{C}$, BMPs gradually increase in size and form the percolating network, which is the long-range FM phase. In the meantime, the BMPs isolated from the FM network behave like fragmented superparamagnetic (SPM) particles. This picture of the mixed FM and SPM is supported by Monte Carlo simulations ${ }^{20,21}$ and recent experiments, ${ }^{22,23}$ it also successfully explains the high-field resistivity behavior in InMnSb. ${ }^{11}$ This two-phase model makes physical sense due to the inevitable inhomogeneous Mn distribution, which leads to the relatively hole-rich FM regions coexisting with the less hole-rich SPM regions.

Note that even in the SPM phase, there should be a nonzero twofold anisotropy term in MR. The external magnetic field can align the randomly distributed BMP moments. As the sample is rotated in $H$, the aligned moment sees the twofold anisotropy with respect to the electric current. It is analogous to the usual AMR and can also be described by Eq. (1). At $T>T_{C}$, the SPM phase is the only source of the anisotropy in $\rho_{x x}$ and the situation is much simpler. We attempt to analyze the $T>T_{C}$ data since we only have to deal with the SPM component. Note that in Eq. (1), the twofold AMR due to the SPM clusters with moment $\mu$ is propor- tional to the square of the aligned total moment, i.e., $\cos ^{2}\left(\phi_{M}\right)=\left(M / M_{s}\right)^{2}$. For SPM particles, the moment $M$ is described by the Langevin function $M / M_{s}=L(x)=\operatorname{coth} x$ $-1 / x, x$ being $\mu H / k_{B} T$. A decrease in $T$ or an increase in $H$ can enhance the magnetic moment to produce a large MR effect. We fit $A_{u}=P \times\left[L\left(\mu H / k_{B} T\right)\right]^{2}$ to the high-temperature field-dependence data and extract the particle moment $\mu$ and total number of particles, $P$, both of which are displayed in the inset of Fig. 4(a). As $T$ increases, the individual BMP cluster size $(\sim \mu)$ decreases as the carrier-mediated exchange interaction weakens; meanwhile, the total number of BMPs increases. The size of BMPs is estimated to be about $6 \mathrm{~nm}$ at $50 \mathrm{~K}$. A somewhat larger BMP size was reported earlier for $T<T_{C}{ }^{23}$ Since the BMP size decreases rapidly above $T_{C}$, we believe our result is still reasonable.

For $T<T_{C}$, the percolating FM phase starts to contribute to $\rho_{x x}$, which leads to a nonzero $A_{c}$, as shown in Fig. 5. Additionally, the FM phases also contribute to the twofold anisotropy in $\rho_{x x}$, as seen in Eq. (4). Although both FM and SPM phases contribute to the twofold symmetry or $A_{u}$, they have opposite trends in temperature due to the expansion of the FM phase and the shrinkage of the SPM phase at low temperatures. The overall temperature dependence of twofold symmetry depends on the competition between these two phases below $T_{C}$. As a result, a peak in $A_{u}$ appears at the intermediate temperatures, as shown in Fig. 5. The peak position shifts to higher temperatures as the field strength increases because the applied field enhances the contribution from the SPM phase at high temperatures. Here, we do not attempt to fit the resulting temperature dependence of $A_{u}$ for lack of detailed knowledge of the two phases below $T_{C}$. $A_{c}$ is suppressed by a magnetic field at all temperatures. We do not have a satisfactory explanation for this field effect.

In summary, we have found a strong magnetocrystalline anisotropic resistance effect in epitaxial GaMnAs films. The fourfold term in longitudinal magnetoresistance is related to the long-range FM order at $T<T_{C}$, whereas the twofold term originates from both FM and SPM spins. This effect may be used in investigating ferromagnetic ordering mechanism in other dilute magnetic semiconductors and ferromagnetic oxides.

The work at UCR was supported in part by CNID and the work at UCSB was supported in part by CNID, the ONR, and the AFOSR. D.W., P.W., and J.S. would like to thank Y. $\mathrm{Pu}$ for his technical assistance. D.W. acknowledges the partial support by the National Basic Research Program of China (2007CB925104).
${ }^{1}$ T. R. Mcguire and R. I. Potter, IEEE Trans. Magn. MAG-11, 1018 (1975).

${ }^{2}$ U. Ruediger, J. Yu, S. Zhang, A. D. Kent, and S. S. P. Parkin, Phys. Rev. Lett. 80, 5639 (1998).

${ }^{3}$ H. X. Tang, S. Masmanidis, R. K. Kawakami, D. D. Awschalom, and M. L. Roukes, Nature (London) 431, 52 (2004).
${ }^{4}$ Yong Pu, E. Johnston-Halperin, D. D. Awschalom, and Jing Shi, Phys. Rev. Lett. 97, 036601 (2006).

${ }^{5}$ T. Jungwirth, J. Sinova, K. Y. Wang, K. W. Edmonds, R. P. Campion, B. L. Gallagher, C. T. Foxon, Q. Niu, and A. H. Macdonald, Appl. Phys. Lett. 83, 320 (2003).

${ }^{6}$ R. P. van Gorkom, J. Caro, T. M. Klapwijk, and S. Radelaar, 
Phys. Rev. B 63, 134432 (2001).

${ }^{7}$ K. Y. Wang, K. W. Edmonds, R. P. Campion, L. X. Zhao, C. T. Foxon, and B. L. Gallagher, Phys. Rev. B 72, 085201 (2005).

${ }^{8}$ W. Limmer, M. Glunk, J. Daeubler, T. Hummel, W. Schoch, R. Sauer, C. Bihler, H. Huebl, M. S. Brandt, and S. T. B. Goennenwein, Phys. Rev. B 74, 205205 (2006).

${ }^{9}$ T. Hayashi, Y. Hashimoto, S. Katsumoto, and Y. Iye, Appl. Phys. Lett. 78, 1691 (2001).

${ }^{10}$ T. Omiya, F. Matsukura, T. Dietl, Y. Ohno, T. Sakon, M. Motokawa, and H. Ohno, Physica E (Amsterdam) 7, 976 (2000).

${ }^{11}$ M. Csontos, T. Wojtowicz, X. Liu, M. Dobrowolska, B. Janko, J. K. Furdyna, and G. Mihaly, Phys. Rev. Lett. 95, 227203 (2005).

${ }^{12}$ H. Ohno, H. Munekata, T. Penney, S. von Molnar, and L. L. Chang, Phys. Rev. Lett. 68, 2664 (1992).

${ }^{13}$ A. Oiwa, S. Katsumoto, A. Endo, M. Hirasawa, Y. Iye, H. Ohno, F. Matsukura, A. Shen, and Y. Sugawara, Solid State Commun. 103, 209 (1997).

${ }^{14}$ T. Jungwirth, M. Abolfath, J. Sinova, J. Kucera, and A. H. Macdonald, Appl. Phys. Lett. 81, 4029 (2002).

${ }^{15}$ S. T. B. Goennenwein, S. Russo, A. F. Morpurgo, T. M. Klapwijk,
W. VanRoy, and J. DeBoeck, Phys. Rev. B 71, 193306 (2005).

${ }^{16}$ H. X. Tang, R. K. Kawakami, D. D. Awschalom, and M. L. Roukes, Phys. Rev. Lett. 90, 107201 (2003).

${ }^{17}$ R. R. Birss, Symmetry and Magnetism (North-Holland, Amsterdam, 1964).

${ }^{18}$ U. Ruediger, J. Yu, S. Zhang, A. D. Kent, and S. S. P. Parkin, Phys. Rev. Lett. 80, 5639 (1998).

${ }^{19}$ A. W. Rushforth, K. Výborný, C. S. King, K. W. Edmonds, R. P. Campion, C. T. Foxon, J. Wunderlich, A. C. Irvine, P. Vašek, V. Novák, K. Olejník, Jairo Sinova, T. Jungwirth, and B. L. Gallagher, Phys. Rev. Lett. 99, 147207 (2007).

${ }^{20}$ R. N. Bhatt, M. Berciu, M. P. Kennett, and X. Wan, J. Supercond. 15, 71 (2002).

${ }^{21}$ A. Kaminski and S. Das Sarma, Phys. Rev. Lett. 88, 247202 (2002).

${ }^{22}$ K. Hamaya, T. Koike, T. Taniyama, T. Fujii, Y. Kitamoto, and Y. Yamazaki, Phys. Rev. B 73, 155204 (2006).

${ }^{23}$ K. Hamaya, T. Taniyama, Y. Kitamoto, T. Fujii, and Y. Yamazaki, Phys. Rev. Lett. 94, 147203 (2005). 